(but not a deletion mutant lacking the ATP binding site) can specifically bind to ATP analogues. Although nobody has yet shown that ATP hydrolysis occurs, it seems likely that CED-4 acts as a context-dependent ATPase. By analogy with the heat shock proteins, it could be that CED-4 is a proCED-3specific chaperone, stabilizing and regulating the inactive proform. ATP hydrolysis, which might normally be inhibited by CED-9 binding, could provide the energy necessary for a conformational change and autocatalytic activation of CED-3.

Is any of this information relevant to caspase activation in mammals? The first hint that it is came from the observation by Chinnaiyan et al. ${ }^{9}$ that $\mathrm{Bcl}-\mathrm{x}_{\mathrm{L}}$ will readily associate with caspase- 1 when the two proteins are coexpressed in mammalian cells, even though they show no affinity for each other in vitro. The authors suggested that an endogenous, CED-4-like activity (and thus a mammalian apoptosome) exists in mammals that could act as a bridge between $\mathrm{Bcl}-\mathrm{x}_{\mathrm{L}}$ and caspase- 1 .

Even more dramatic evidence for a worm-mammal connection has been unearthed by Zou et al. ${ }^{2}$. This group had previously $^{10}$ developed an extract system derived from human cells, that, upon the addition of deoxyribose-ATP, would result in the proteolytic activation of caspase-3. Purification of the components in the extract required for this activity led to the isolation of three apoptosis protease-activating factors (Apafs), which in combination seem to be sufficient to promote caspase- 3 processing upon addition of deoxyribose-ATP. So, at least functionally, the Apafs are the mammalian equivalent of C. elegans CED-4. Rather surprisingly, Apaf-2 is cytochrome $c$, the ubiquitous protein involved in the mitochondrial electron transport chain ${ }^{10}$. The counterintuitive corollary of this observation is that, in cells doomed to die, cytochrome $c$ must leave the mitochondria for the cytosol (where the caspases reside). Counterintuitive this may be, but it appears to be true, at least in mammalian cells (reviewed in refs 5, 11).

In their paper, Zou et al. ${ }^{2}$ present the sequence of Apaf-1, a protein of $M_{\mathrm{r}} 130,000$. Remarkably, part of it shows a striking similarity to that of CED-4, with the two proteins aligning over most of the CED-4 sequence, including the nucleotide binding site. Flanking the CED-4 homology region are two other domains that provide additional hints as to how the Apafs might direct caspase activation (Fig. 1b). The amino terminus of Apaf-1 shows some sequence similarity to the prodomain of CED-3, and probably constitutes a caspase-recruitment (CARD) domain. CARD domains are found in a number of cell death proteins (including CED-3 and CED-4) and may bind directly to caspases $^{12}$. This domain might thus be the link between the Apafs and caspase-3. At the carboxy terminus of the protein is a large domain containing $12 \mathrm{WD}-40$ repeats. Such repeats usually mediate protein-protein interactions, and could in this case be involved in the physical interaction that Zou et al. ${ }^{2}$ observed between Apaf-1 and Apaf-2/cytochrome $c$.

So what does it all mean? That both CED4 and Apaf- 1 promote the activation of caspases suggests that their molecular mechanism of action is likely to be the same. However, as is the case with CED-9 versus Bcl-2, and CED-3 versus the caspases, regulation of Apaf-1 is likely to be more complex (or sophisticated, depending on your point of view), not least because mammalian cells must integrate many more signals before deciding whether to live or die.

Plenty of issues remain. Is Apaf-1, like CED-4, regulated by interaction with $\mathrm{Bcl}-2$ family members? If so, does it show any preferences in its dealings with the various factions within the family? What are we to make of the WD-40 repeats in Apaf-1, and of their absence in CED-4? If they bind to cytochrome $c$ (and possibly Apaf-3), does binding result in activation, or in relief of an intrinsic inhibitory activity? And are we to conclude that, in C. elegans, cytochrome $c$ is not involved in cell death?

Finally, does Apaf-1 constitute the only mechanism that leads to caspase- 3 activation? In C. elegans, this is clearly the case, because in the absence of CED-4 there is no cell death whatsoever. Such a dramatic effect seems unlikely in mammals, be it because of alternative activation pathways or redundancy with other caspases. Indeed, what about the other caspases? Do they each have their own Apaf-1 homologue, do they share a common activator, or are they simply activated by other caspases (through the fabled caspase cascade)? Questions, questions much work remains to be done.

Michael O. Hengartner is at the Cold Spring Harbor Laboratory, 1 Bungtown Road, Cold Spring Harbor, New York 11724, USA.

e-mail:hengartn@cshl.org

1. Hengartner, M. O. \& Horvitz, H. R. Curr. Opin. Gen. Dev. 4 581-586 (1994).

2. Zou, H., Henzel, W. J., Liu, X., Lutschg, A. \& Wang, X. Cell 90, 405-413 (1997).

3. Chinnaiyan, A. M., Chaudhary, D., O'Rourke, K., Koonin, E. V. \& Dixit, V. M. Nature 388, 728-729 (1997).

4. Seshagiri, S. \& Miller, L. K. Curr. Biol. 7, 455-460 (1997).

5. Shaham, S. \& Horvitz, H. R. Genes Dev. 10, 578-591 (1996).

6. Jacobson, M. D. Curr. Biol. 7, R277-R281 (1997).

7. Reed, J. C. Nature 387, 773-776 (1997).

8. James, C., Gschmeissner, S., Fraser, A. \& Evan, G. I. Curr. Biol. 7, 246-252 (1997)

9. Chinnaiyan, A. M., O’Rourke, K., Lane, B. R. \& Dixit, V. M. Science 275, 1122-1126 (1997).

10. Liu, X., Kim, C. N., Yang, J., Jemmerson, R. \& Wang, X. Cell 86, 147-157 (1996).

11. Golstein, P. Science 275, 1081-1082 (1997)

12. Hofmann, K. et al. Trends Biochem. Sci. 22, 155-156 (1997).
$\mathrm{O}$

icroporous solids Cobalt caged for catalysis transition metals. They demonstrate it by synthesizing cobalt phosphates and cobalt metal phosphates, and the family of materials that can be made in his way should include a large variety of compositions and crystalline structures, with the potential for new catalytic reactions

In most cases, the new cobalt phosphate structures have been observed before in the aluminosilicate zeolites, a class of materials that are not only synthesized industrially as catalysts and adsorbents, but also formed in nature by the weathering of volcanic rock and marine sediments. Similar structural mimicry of these naturally occurring minerals has been observed before in beryllium ${ }^{2}$ and zinc ${ }^{3}$ phosphate frameworks, but its extension into the transition elements is of interest because of their greater variety of useful chemical properties. With many oxidation states so easily accessible, transition metals will allow a wider range of reactions than the solid-acid chemistry of traditional zeolites - they should be able to catalyse oxidation and reduction reactions for example. The magnetic behaviour of the transition

metals might also be exploited to create new types of molecule-selective devices.

The commercial applications of microporous materials are continually expanding. Materials of this type are often referred to as frameworks, to describe their scaffold-like, rigid crystalline structures; or as molecular sieves, because their pore cross-sections are of molecular dimensions. As well as the aluminosilicate zeolites, there are titanium silicates, aluminium phosphates, metal sulphides and selenides, transition-metal oxides, and many others.

These materials are used as catalysts in refining gasoline and diesel fuel; they aid powdered laundry detergents by softening water; and they can efficiently separate air into its valuable pure components, $\mathrm{N}_{2}$ and $\mathrm{O}_{2}$. Newer applications include the use of the molecule-sized cavities within these materials to carry out the selective oxidation of simple organic compounds into useful chemicals ${ }^{4}$. Microporous oxides, used as ion exchangers to selectively trap the highly radioactive isotopes ${ }^{137} \mathrm{Cs}$ and ${ }^{90} \mathrm{Sr}$, are likely to play a leading role in cleaning up the nuclear waste generated by weapons programmes and nuclear power generation ${ }^{5,6}$.

The challenge in synthesizing new frame- 


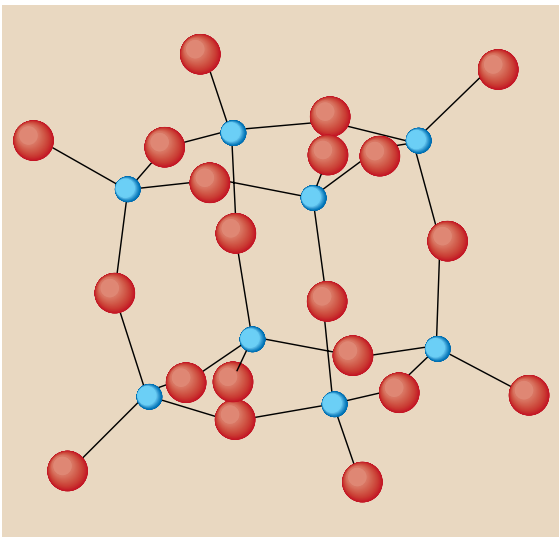

Figure 1 This hypothetical structure was proposed for an aluminosilicate zeolite ${ }^{7}$, but has only now been realized as a cobalt phosphate. The new material has cobalt, aluminium and phosphorus at the blue sites, and oxygen at the red sites. The method of synthesis should yield a great number of new microporous materials, opening up a wider range of chemistry for these open framework catalysts.

works is to find a recipe that at least partially dissolves and transports the components in the reaction solvent, and also causes a microporous framework to form rather than an undesirable non-porous structure. The significance of the new phases ${ }^{1}$ lies in their open structures (including one novel form) and their compositions, and in the discovery of a new synthesis procedure that made these materials possible.

The authors chose a clever combination of a new cobalt reagent, $\mathrm{CoCO}_{3}$, and a largely non-aqueous ethylene glycol solvent system to create conditions that lead to the ready synthesis of the cobalt phosphates. The pores within the crystallized products are filled with organic ammonium cations and water, which stabilize the structure during crystallization by space filling and charge compensation. But whether this metal carbonate route is more generally applicable to other divalent transition metals is not yet clear.

Another intriguing aspect of the cobalt

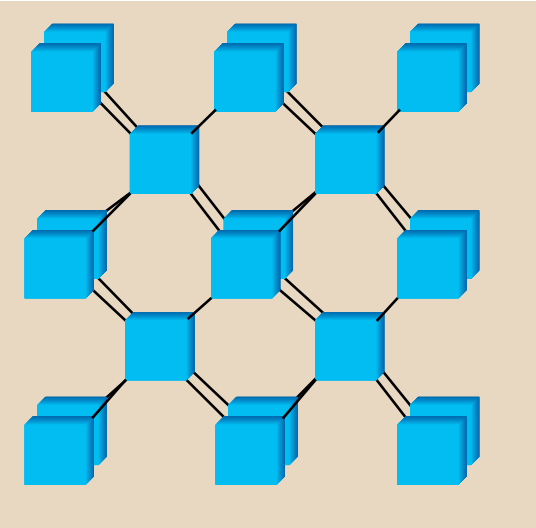

Figure 2 Cubic framework of the building block in Fig. 1. The pseudo-octagonal micropores in this structure are $3.8 \AA$ in diameter and permeate the structure in three dimensions. phosphates is the formation of an unprecedented structure that Stucky and colleagues have called ACP-1, for aluminium cobalt phosphate 1 (Fig. 1). This structure was erroneously proposed for one of the synthetic aluminosilicate zeolites, known as cubic zeolite $\mathrm{P}$, by R. M. Barrer et al. ${ }^{7}$, in 1959. Nearly 40 years have passed between Barrer's proposal and the successful synthesis of the hypothetical structure, albeit in a completely different composition. The ACP-1 structure is an array of nine cube-shaped building blocks arranged at the corners and in the centre of a larger cube. Repeating this structure produces a framework that contains a three-dimensional network of 3.8-Å channels (Fig. 2).

A key question that always arises when a new family of frameworks is discovered is whether the latent microporosity of the materials can be accessed. The porosity remains latent until a method is devised to remove the space-filling, chargecompensation molecules without destroy- ing the framework. Without the removal of this 'stuffing', much of the promise of novel or improved applications is diminished. Achieving access is a crucial step in the application of the cobalt phosphates - if successful it will allow study of the redox chemistry of the frameworks and of their potential as selective oxidation catalysts. The cobalt phosphates may also be good at adsorptive separations of gases and ion exchange processes.

Robert L. Bedard is at the UOP Research Center, 50 East Algonquin Road, Des Plains, Illinois 60017-5016, USA.

\footnotetext{
1. Feng, P., Bu, X. \& Stucky, G. Nature 388, 735-741 (1997).

2. Harvey, G. \& Meier, W. M. in Studies in Surface Science and Catalysis 49a, (eds Jacobs, P. A. \& Van Santen, R. A.) 411-420 (Elsevier, Amsterdam, 1989).

3. Gier, T. E. \& Stucky, G. D. Nature 349, 508-510 (1991).

4. Neri, C., Buonomo, F. \& Anfossi, B. EU Pat. 100118 (1985).

5. Anthony, R. G., Philip, C. V. \& Dosch, R. G. Waste Management 13, 503-512 (1993)

6. Zheng, Z. et al. Ind. Eng. Chem. Res. 35, 4246-4256 (1996).

7. Barrer, R. M., Bultitude, F. W. \& Kerr, I. S. J. Chem. Soc. 1521-1528 (1959).
}

\section{A silent channel opens its gates}

\section{Peter H. Seeburg}

$\mathrm{M}$ any human neurodegenerative diseases, such as amyotrophic lateral sclerosis (also known as Lou Gehrig's disease), Alzheimer's disease, Parkinson's disease and Huntington's chorea, lead to the demise of distinct nerve-cell populations. Yet in no case is the selective vulnerability of the affected cells, nor the underlying damaging principle, understood.

A popular view is that these sinister diseases are caused - or at the very least exacerbated - by the unphysiological activity of cationselective membrane channels operated by glutamate, which is the most abundant excitatory neurotransmitter in the brain. Now, the discovery by Zuo et al. ${ }^{1}$ (page 769 of this issue) provides the first direct evidence in support of this view. The authors have traced the selective neurodegeneration that occurs in a spontaneous neurological mouse mutant (lurcher) to the constitutive activation of a member of the glutamate-receptor-channel family.

First described in 1960, the lurcher $(L c)$ mouse derives its name from a peculiar gait that is characteristic of heterozygous carriers of the mutant gene. These mice develop a lack of balance (ataxia), because all of the Purkinje cells in the cerebellum - a brain structure that is important for motor coordination - are lost. Mice that are homozygous for the $L c$ gene die shortly after birth, due to a massive loss of hindbrain neurons during embryonal development. These neurons include the trigeminal motor nucleus, which controls the muscles required for suckling. So, the neurological phenotype of the lurcher gene is characterized by selective neuronal cell death. In a collaborative effort ${ }^{1}$ between the labs of Nathaniel Heintz and David J. Linden, the molecular cause for this neuronal death has now been unravelled.

Ever since John Olney and Lawrence Sharpe ${ }^{2}$ reported that infant rhesus monkeys develop brain lesions when exposed to glutamate, attention has been directed towards how the abundant excitatory neurotransmitter L-glutamate might become a culprit in acute and degenerative cell death in the brain. In an elegant series of experiments, Dennis $\mathrm{Choi}^{3}$ and colleagues showed that this glutamate-evoked cell death can be mimicked in vitro, and that the onset and extent of cell death are critically dependent on the influx of $\mathrm{Ca}^{2+}$ into neurons.

Our mechanistic understanding of these observations was boosted by the molecular characterization of the various receptor channels that are activated by L-glutamate in central neurons ${ }^{4}$. These cation-selective channels are composed of sequence-related subunits. They form a family of glutamate receptors (GluRs)which have distinct properties, commensurate with their particular roles in synaptic physiology. The best-characterized GluRs are the AMPA (aminohydroxymethylisoxalate propionic acid) and NMDA ( $N$-methyl-D-aspartate) receptor channels, which mediate fast excitatory neurotransmission.

Whereas most AMPA receptors are permeable only to monovalent cations, NMDA receptors are designed for the controlled synaptic influx of $\mathrm{Ca}^{2+}$ ions. Most neurons carry both types of GluR, not only in synapses 